\title{
Review of: "High-Speed Imaging Reveals the Bimodal Nature of Dense Core Vesicle Exocytosis and Jet Flow through the Fusion Pore"
}

\author{
Claudia Tomes ${ }^{1}$ \\ 1 Universidad Nacional de Cuyo
}

Potential competing interests: The author(s) declared that no potential competing interests exist.

This is an invited commentary about the article "High-Speed Imaging Reveals the Bimodal Nature of Dense Core Vesicle Exocytosis and Jet Flow through the Fusion Pore", authored by Pengcheng Zhang, David Rumschitzki and Robert H. Edwards

The paper describes a high-speed imaging protocol to reveal fusion pore characteristics during DCV exocytosis in primary mouse adrenal chromaffin cells. The Introduction addresses what is known/inferred regarding the heterogeneity of exocytotic responses in neuroendocrine cells and sets up the framework to investigate what is not known. The authors make a good case at comparing the strengths and limitations of TIRF with conventional fluorophores and amperometric recordings and at how the read outs of both methods are not easy to integrate or reconcile. The regulation of secretion by the fusion pore itself was, until now, assigned to size, behavior as a sieve, and commitment to full fusion versus kiss and run. The findings reported here by Zhang et al add a layer of complexity by revealing that the duration of the fusion pore is bimodal and regulated rather than stochastic, as previously assumed. In doing so, this paper opens the door to future work on the molecular mechanisms that underlie the bimodal nature of regulated DCV exocytosis.

The experiments are elegant and very meticulous, many possible hypotheses and interpretations are offered and justified. Each conclusion is supported by more than one experimental approach and by numerous, rigorous, original controls.

Interpretation of the results includes deep insights in addition to the primary description, such insights may or may not have been contemplated when outlining the experiments, but there they are, exquisitely capitalising on the findings. Examples that illustrate this point are: i) slow events derive exclusively from docked vesicles, which means that the state of docking influences subsequent behavior of the pore. ii) Release by newcomers is always fast, which implies that tethering, priming, docking and fusion occur within a few milliseconds of granule arrival at the fusion site. iii) Rapid efflux of luminal cargo through a narrow pore delays external dye entry, and so on and so forth.

If I may make a few suggestions, the TIRF images in Figure 1 are a bit small to appreciate clearly. Figure 1 -figure supplement 3: I am not entirely sure of what panel B shows. Results shown in Fig. $2 \mathrm{H}$ are not 
described. Unfortunately I couldn't find the videos.

There are a few phrases that I had trouble understading, such as:

- "...the fluorescence should not increase with excitation by a field of uniform strength" (page 9, line 14).

- Legend to Figure 4: "the prolonged exposure time required for dual imaging of BDNF-pHluorin and FFN limited capture of the FFN event to a single (D and G) or no (F) frame"

- Page 9: Was swelling ruled out? How? I find it hard to connect the last sentence on that page $3 / 4$ "Taken together, these observations suggest that the fusion pore opens at the onset of fluorescence increase for slow as well as fast events" $3 / 4$ to the premisses stated above it.

- Page 13, lines 19-21: “We also observe very few vesicles arriving at the plasma membrane that do not undergo fusion, consistent with a large population of stably docked granules". I am not sure about what "consistent" indicates here. Did stably docked granules arrived when conditions were not suitable for release?

- Page 14, lines 12-17: “If chromaffin granules swell upon exocytosis and membrane fusion precedes swelling, as reported for mast cell as well as chromaffin granules (Breckenridge and Almers, 1987; Terakawa et al., 1991), then swelling would account for the increase in FFN fluorescence observed by confocal as well as TIRF microscopy and hence independent of the evanescent field. In this case, membrane fusion occurs no later than the onset of FFN fluorescence increase, well before Alexa dye entry". This text is a bit confusing for me. If fusion precedes swelling, FFN fluorescence will increase by both phenomena within the TIRF field and concomitantly with Alexa. If swelling precedes fusion, then FFN fluorescence will be detected by both TIRF and confocal microscopy before Alexa entry. I am not sure what "In this case," in the last sentence refers to.

Pointing out these few concepts that I found hard to grasp does not in the least diminish the excellent rating I would give this paper. I can't wait to see what the authors will do next with the new imaging technique reported here. 